\title{
Molecular Mechanisms and Therapeutic Strategies of Chronic Renal Injury: Physiological Role of Angiotensin II-Induced Oxidative Stress in Renal Medulla
}

\author{
Takefumi Mori ${ }^{1, *}$, Allen W. Cowley, Jr. ${ }^{2}$, and Sadayoshi Ito ${ }^{1}$ \\ ${ }^{I}$ Division of Nephrology, Endocrinology and Vascular Medicine, Tohoku University Graduate School of Medicine, \\ Sendai 980-8574, Japan \\ ${ }^{2}$ Department of Physiology, Medical College of Wisconsin, Milwaukee, WI 53226, USA
}

Received September 28, 2005; Accepted November 7, 2005

\begin{abstract}
Renal medullary circulation has now been found to play a fundamental role in regulating long-term blood pressure control and fluid balance. Elevation of superoxide or reduction of nitric oxide (NO) in renal medulla decreases medullary blood flow and $\mathrm{Na}$ excretion, resulting in sustained hypertension. Angiotensin II (Ang II)-induced interaction of superoxide and NO was determined in thin tissue strips isolated from the renal outer medullary region of Sprague-Dawley rats using fluorescent microscopy techniques. Ang II can induce diffusion of NO, but not superoxide, from the medullary thick ascending limb (mTAL) to the surrounded vasa recta. However, when NO is reduced by the NO scavenger carboxy-PTIO, Ang II can induce superoxide diffusion from mTAL to vasa recta pericytes. Therefore, the physiological action of oxidative stress in renal medullary region is demonstrated as balance of superoxide and NO diffusion ("tubulo-vascular cross-talk"). These results explain how chronically hypoxic medulla can maintain blood flow. In other studies using chronically instrumented rats, we found that nearly $70 \%$ of Ang II-induced medullary renal injury was dependent on pressure determined by servo-control of renal perfusion pressure, whereas $30 \%$ of the injury was non-hemodynamic. We conclude that oxidative stress within the renal medulla can induce hypertension and also make the kidney functionally more vulnerable to the effects of Ang II.
\end{abstract}

Keywords: angiotensin II, oxidative stress, reactive oxygen species, hypertension, renal injury

\section{Introduction}

Although renal medullary blood flow (MBF) is supplied by only $5-10 \%$ of the total renal flow, it has been found to play an important physiological role in the $\mathrm{Na}$ homeostasis and long-term arterial blood pressure (1). Also only a $15-30 \%$ reduction of MBF can result in decrease of $\mathrm{Na}$ excretion and sustained hypertension (1). This is not mediated by the well-known countercurrent mechanisms involved largely in processes of urine concentration, but by unique mechanisms discussed below. Although the $\mathrm{PO}_{2}$ level of renal medulla is less than $40 \%$, the active tubular transport requires oxygen,

*Corresponding author. tmori2i@mail.tains.tohoku.ac.jp

Published online in J-STAGE: January 11, 2006

DOI: 10.1254/jphs.FMJ05003X2 especially in the region of the medullary thick ascending limb (mTAL) $(1,2)$. Since oxygen utilization in the outer medulla is flow limited, it appears that tubules in this region have acquired a unique mechanism using free radicals in response to vasoconstrictors to protect tubular cells from hypoxia. In this brief review, we therefore focus on this mechanism that participates in the pathophysiological role of angiotensin II (Ang II) in the renal medullary circulation and renal injury.

\section{Basics of medullary circulation to the control of} blood pressure

MBF is mostly supplied from the efferent arterioles of juxtamedullary glomeruli and radiated into descending vasa recta bundles in the outer medulla. The descending vasa recta enter straight into the inner medulla and loop 
at the tip of the papilla flowing to ascending vasa recta $(1,2)$. Even with the anatomical relationships between $\mathrm{MBF}$ and cortical blood flow (CBF), a number of studies have shown that MBF can be regulated independently of CBF $(1-12)$. The incongruity of medullary flow was demonstrated by the technique in which vasoactive substances were delivered into the renal medullary interstitial space with a small implanted catheter and the flow measured with implanted optical fibers and laser-Doppler flowmetry techniques, as shown in Fig. 1 $(1-12)$. The most prominent changes in MBF was seen when the nitric oxide synthase (NOS) inhibitor $N^{\mathrm{G}}$-nitroL-arginine methylester (L-NAME) was infused into the medullary interstitial space. Infusion of L-NAME $(120 \mu \mathrm{g} / \mathrm{h})$ into this region of anesthetized SpragueDawley (SD) rats reduced MBF by nearly $70 \%$ of the

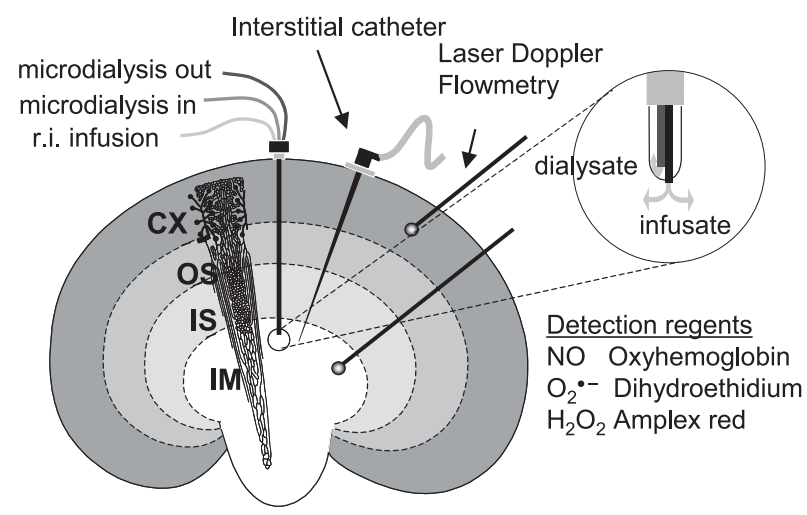

\begin{tabular}{|c|c|c|c|c|c|}
\hline$\underline{\text { Infusion }}$ & microdialysis & $\underline{\text { MBF }}$ & $\underline{\text { CBF }}$ & $\underline{\text { UNa }}$ & BP \\
\hline L-NAME, r.i.(SD) & NO $\downarrow$ & $\downarrow$ & - & $\downarrow$ & $\uparrow$ \\
\hline Ang II, i.v.(SD) & NO $\uparrow$ & - & - & & - \\
\hline Ang II, i.v.+L-NAME, r.i. (S & SD) NO - & $\downarrow$ & - & & $\uparrow$ \\
\hline DETC, r.i. & $\mathrm{O}_{2} \cdot-\uparrow$ & $\downarrow$ & - & $\downarrow$ & $\uparrow$ \\
\hline $\mathrm{H}_{2} \mathrm{O}_{2}$, r.i. & $\mathrm{H}_{2} \mathrm{O}_{2} \uparrow$ & $\downarrow$ & - & $\downarrow$ & $\uparrow$ \\
\hline Ang II, i.v.(DahIS) & NO - & - & - & & $\uparrow$ \\
\hline Ang II, i.v.(BN) & NO $\uparrow$ & - & - & & $\uparrow$ \\
\hline Captopril, r.i.(SHR) & & $\uparrow$ & - & $\uparrow$ & $\downarrow$ \\
\hline
\end{tabular}

Fig. 1. Tools to examine renal medullary circulation and medullary function in vivo. An interstitial catheter was implanted to deliver drugs to the renal medulla. A laser-Doppler flowmetry technique with optical fibers implanted in the cortex and medulla measured regional blood flow. The renal microdialysis technique was performed to detect changes in reactive oxygen and nitrogen species from the renal interstitial fluid. The lower table shows the summary of the results from the published studies. Figure modified from Ref. 1 with permission. SD: Sprague-Dawley rats, DahlS: Dahl saltsensitive rats, BN: Brown-Norway rats, SHR: spontaneously hypertensive rats. i.v.: intravenous infusion, r.i.: renal interstitial infusion. MBF: medullary blood flow, CBF: cortical blood flow, UNa: urinary $\mathrm{Na}$ excretion, BP: blood pressure. CX: cortex, OS: outer stripe of outer medulla, IS: inner stripe of outer medulla, IM: inner medulla. baseline without altering CBF and decreased Na excretion by $34 \%$ (3). In other studies where L-NAME was infused intravenously for one week at low doses, it was found that there was a selective reduction of medullary flow in the absence of any measurable changes of cortical blood flow, resulting in sodium retention and hypertension (4). These results indicate that MBF can be regulated independently of $\mathrm{CBF}$ and that $\mathrm{NO}$ can be one of the specific modulators of MBF.

Evidence that vasa recta can constrict to regulate MBF independent of CBF has been shown by several investigators $(13,14)$. Descending vasa recta are unique capillaries that have a layer of endothelium and some surrounded pericytes, which is the smooth muscle remnant. Park et al. have shown that pericytes of descending vasa recta contain alpha smooth muscle actin, suggesting that it vasoconstricts similar to smooth muscle cells (13). Pallone and colleagues have shown using microdissected perfused vasa recta from rat outer medulla that these capillaries can indeed constrict and dilate in response to several substances including Ang II and NO (14). Interestingly, descending vasa recta constricted at the points where the foot process of pericytes surrounded the capillaries (14).

The importance of the fundamental role of pressurenatriuresis in the regulation of blood pressure and fluid volume was discovered by Guyton and associates (15). It has been demonstrated that pressure natriuresis is blunted in hereditary and secondary forms of hypertension in both animal models and humans (16). It has also been shown that the medullary circulation could largely contribute to this mechanism $(1,16)$. A series of experiments done by Roman and Cowley have demonstrated that renal interstitial hydrostatic pressure (RIHP) can be importantly influenced by medullary blood flow $(1,16)$. Increases in renal perfusion pressure in anesthetized rats are followed by elevations of MBF as determined by small implanted optical fibers and laserDoppler flowmetry techniques (5). These studies have shown that MBF has weaker autoregulation than CBF. The pressure-induced increases of MBF were also accompanied by increases in RIHP and urinary $\mathrm{Na}$ excretion (17). From these observed relationships, it has been proposed that this lack of autoregulation of medullary blood flow serves as the trigger for the pressure-natriuresis mechanism whereby higher renal perfusion pressure increases MBF that in turn elevates RIHP and thereby reduces $\mathrm{Na}$ reabsorption.

\section{Physiological role of Ang II in medullary circulation}

Ang II-induced NO production and associated changes in MBF have been studied in acute anesthetized (6) and 
chronic conscious (7) rats. Zou et al. have shown using a small implanted optical fiber in the kidney and the laser-Doppler flowmetry technique that a small subpressor dose of Ang II $\left(5 \mathrm{ng} \cdot \mathrm{kg}^{-1} \cdot \mathrm{min}^{-1}\right)$ infused intravenously to anesthetized $\mathrm{SD}$ rats did not alter $\mathrm{MBF}$, $\mathrm{CBF}$, or medullary tissue $\mathrm{PO}_{2}$ (6). However when LNAME was infused into the medullary interstitial space at a dose that did not alter baseline MBF, the same dose of intravenous Ang II infusion reduced MBF and lowered medullary $\mathrm{PO}_{2}$ without altering $\mathrm{CBF}$ (6). Medullary NO production using the oxyhemoglobin trapping microdialysis technique was found to be increased by Ang II. These elevations of NO were responsible for maintaining a constancy of MBF in the face of the elevations of circulating Ang II since in rats pretreated with L-NAME, the same dose of Ang II resulted in nearly a $30 \%$ reduction of MBF. These results indicate that Ang II stimulated NO can buffer the reduction of $\mathrm{MBF}$ and protect the renal medulla from hypoxia (6). Optical fibers were also implanted chronically into the interstitial space of SD rats and local blood flow was determined by laser-Doppler flowmetry (7). Chronic intravenous infusion of Ang II at a dose that did not increase blood pressure acutely resulted in sustained reductions of MBF, leading to hypertension when a low subpressor dose of L-NAME was administered into the medullary interstitial space (7).

Direct evidence that renal oxidative stress would reduce $\mathrm{MBF}$ and produce sustained hypertension has also been shown. Zou et al. has found that increase in renal superoxide with infusion of SOD inhibitor diethyldithiocarbamic acid (DETC) into renal medullary interstitial space of anesthetized SD rats could reduce MBF and $\mathrm{Na}$ excretion without altering $\mathrm{CBF}$ (8). In contrast, infusion of the SOD mimetic 4-hydroxytetramethylpiperidine-1-oxyl (TEMPOL) into the same region increased MBF and Na excretion in anesthetized SD rats (8), indicating that superoxide is also responsible for the physiological function of the medulla (8). This was confirmed with a chronic rat study done by Makino et al. who showed that long-term administration of DETC into the renal medullary region from small catheters chronically implanted in the medulla of SD rats resulted in specific reduction of MBF and sustained hypertension (9). In this model, increased interstitial superoxide concentrations were determined to ascertain the effect of DETC by micordialysis of the medullary interstitial fluid with the conversion of dihydroethidium to ethidium (9).

Other reactive oxygen species such as hydrogen peroxide $\left(\mathrm{H}_{2} \mathrm{O}_{2}\right)$ have also been found to regulate MBF $(10$, 11). $\mathrm{H}_{2} \mathrm{O}_{2}$ is transformed from superoxide by SOD and scavenged by catalase. Experiments by Chen et al. have demonstrated that infusion of $\mathrm{H}_{2} \mathrm{O}_{2}$ to renal medulla from a small implanted catheter reduced MBF and urinary $\mathrm{Na}$ excretion in anesthetized rats (10). Importantly, when $\mathrm{H}_{2} \mathrm{O}_{2}$ was chronically infused directly into the renal medulla, chronic hypertension resulted that was associated with a 3-fold increase of medullary interstitial $\mathrm{H}_{2} \mathrm{O}_{2}$ level determined by the renal microdialysis methods (11). Taken together, these results demonstrate that superoxide production in the renal medulla can play both a physiological and pathphysiological role in regulating MBF and in the long-term control of blood pressure. The next question that was addressed was whether Ang II-induced oxidative stress could indeed modify MBF and thereby chronically influence renal function and arterial pressure.

\section{Ang II-induced diffusion of free radicals in renal medulla}

From the observations obtained from the microdialysis measurement of NO in the interstitial fluid, it was hypothesized that NO could be diffusing through the kidney and act as a paracrine factor between the cell types. Therefore, the source and the diffusion capability of free radicals in response to Ang II were determined in the medullary region. To accomplish this task, we established a technique that could visualize the real-time increase of free radicals in the living tissue isolated from the renal medulla $(18,19)$. The tissue was freshly isolated from the renal medulla of SD rats after blood was removed by infusion of physiological solution from the renal artery. The inner stripe of the outer medulla was hand dissected under the stereomicroscope while the tissue was maintained in an ice-cooled chamber. The thin tissue contained a single layer of tubules (mainly mTAL) and vessels (vasa recta) that maintained their normal morphological relationships as shown in Fig. 2 $(18,19)$. To determine free radical interactions of pericytes and mTAL or changes within the vasa recta pericytes alone, thin layers of tissue were isolated. For studies in which pericyte activity was to be studied, we first disrupted vasa recta endothelium with microsphere beads perfused from the renal artery to separate the signal from the endothelium to pericytes $(18,19)$. The tissues were placed in the temperature-controlled chamber on the stage of a fluorescent microscope after loading with the free radical fluorescent indicators. DAF-2DA was used for NO detection and dihydroethidium, for superoxide. A ratio-metric measurement was utilized to measure superoxide production by dihydroethidium so that the effects of dye leakage and small changes in focus due to geometric changes would be automatically corrected (19). This became possible 


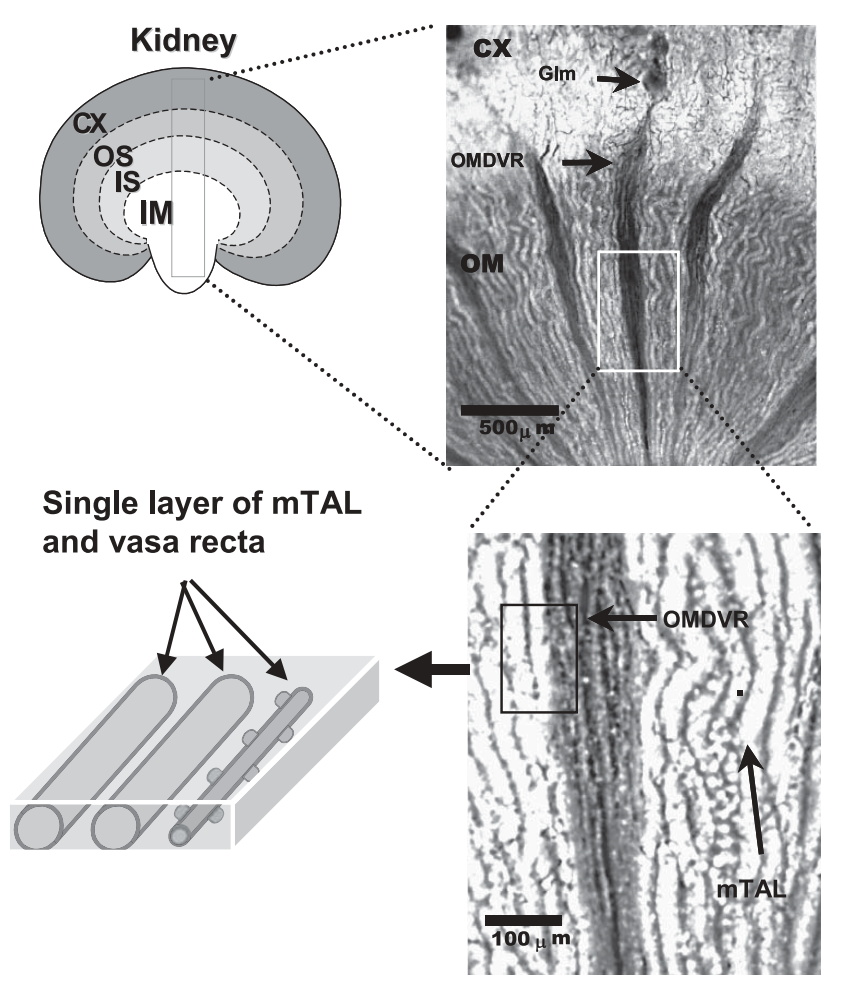

Fig. 2. The technique established for real-time free radical detection in renal medulla. A thin tissue strip was isolated from inner stripe of the outer medulla with morphological characteristics maintained. The photo shows the outer medullary region illuminated by the superoxide indicator dihydroethidium captured under a fluorescent microscope equipped with cooled CCD camera. Photo modified from Ref. 2 with permission. CX: cortex, OS: outer stripe of outer medulla, IS: inner stripe of outer medulla, OM: outer medulla, Glm: glomerulus, IM: inner medulla. OMDVR: outer medullary descending vasa recta, mTAL medullary thick ascending limb.

by using rapid filter changers and a high sensitivity camera. The tissue was stimulated by exchanging the solution in the chamber. By using this technique, we were able to demonstrate the interactions between mTAL and vasa recta pericytes under Ang II stimulation $(18,19)$.

As shown in Fig. 3, $1 \mu \mathrm{mol} / \mathrm{L}$ of Ang II increased NO in pericytes of endothelium disrupted vasa recta when pericytes were present adjacent to the surrounding mTAL (18). However, this dose of Ang II did not increase NO in either pericytes of endothelium disrupted vasa recta or endothelial cells of isolated vasa recta. Significant increases in NO were seen in epithelial cells of mTAL. Since this was the only source of NO production detected within the tissue, these observations demonstrated that the Ang II simulated NO in mTAL that in turn, diffused into adjacent pericytes (18). Interestingly, it was observed that Ang II failed to increase intracellular $\mathrm{Ca}^{2+}$ of vasa recta endothelium and actually
Tubulo-vascular cross-talk

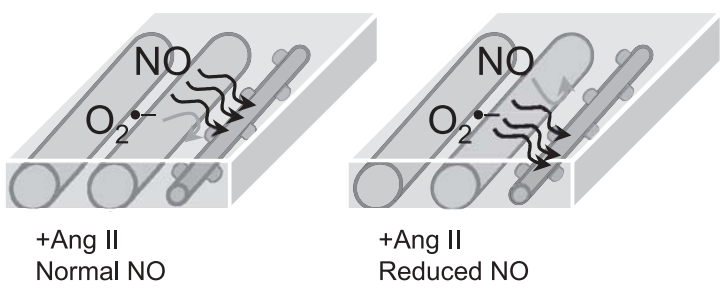

\begin{tabular}{|lccccc|}
\hline Tissue & Stimulation & Preincubation & NO & $\underline{\mathrm{O}_{2}{ }^{\circ-}}$ & $\mathrm{Ca}^{2+}$ \\
pericyte & Ang II & - & - & - & $\uparrow$ \\
pericyte & Ca ionophore & - & - & & $\uparrow$ \\
pericyte with mTAL & Ang II & - & $\uparrow$ & - & \\
pericyte with mTAL & Ang II & C-PTIO & - & $\uparrow$ & \\
pericyte with mTAL & Tempol & - & $\uparrow$ & $\downarrow$ & \\
pericyte with endothelium & Ang II & - & & - & \\
endothelium & Ang II & - & - & $\uparrow$ & $\downarrow$ \\
endothelium & Ca ionophore & - & $\uparrow$ & & $\uparrow$ \\
endothelium & Ang II & Apocynin or DPI & - & \\
mTAL & Ang II & - & $\uparrow$ & $\uparrow$ & $\uparrow$ \\
mTAL & Ang II & C-PTIO & - & $\uparrow$ & \\
mTAL & Ang II & Apocynin or DPI & - & \\
mTAL & Tempol & - & $\uparrow$ & $\downarrow$ & \\
\hline
\end{tabular}

Fig. 3. Schematic summary and results from the experiments performed to demonstrate the diffusion of $\mathrm{NO}$ and superoxide from the medullary thick ascending limb to the adjacent pericytes of the vasa recta. Angiotensin II normally increases diffusion of NO from mTAL to pericytes and serves as tubulo-vascular cross-talk. Superoxide cannot normally diffuse from mTAL to pericytes. However, when NO is reduced by carboxy-PTIO, superoxide can diffuse into the pericytes. C-PTIO: carboxy PTIO, DPI: diphenyleneiodonium, mTAL: medullary thick ascending limb.

decreased $\mathrm{Ca}^{2+}$ levels, as determined by the fluorescent indicator fura-2AM. This then appears to have been responsible for the failure of Ang II to increase NO production in the endothelial cells since when intracellular $\mathrm{Ca}^{2+}$ was increased using a $\mathrm{Ca}$ ionophore, intracellular NO elevations were observed. In contrast, Ang II did not increase in pericytes of vasa recta, even when intracellular $\mathrm{Ca}^{2+}$ was increased using a $\mathrm{Ca}$ ionophore, indicating that pericytes may lack $\mathrm{Ca}^{2+}$ induced NO production (18).

Superoxide production with the same dose of Ang II was also studied. The same dose of Ang II increased superoxide in the endothelium of vasa recta but failed to change the increase in superoxide production in adjacent pericytes (19). Ang II also failed to increase superoxide in vasa recta pericytes in both tissue surrounded by mTAL and isolated vasa recta, while significant increase in intracellular superoxide level was observed in epithelial cells of mTAL (19). However, 
when $\mathrm{NO}$ was reduced by the preincubation of the tissue with the NO scavenger carboxy-PTIO, the same dose of Ang II increased intracellular superoxide in vasa recta pericytes only in tissues surrounded with mTAL (19). Moreover, intracellular NO was increased in vasa recta pericytes surrounded by mTAL when superoxide was scavenged by the SOD mimetic TEMPOL (19).

Taken together, these results indicate that Ang II stimulates both NO and superoxide in epithelial cells of mTAL and that these reactive oxygen species can diffuse into pericytes of vasa recta. In SD rats, it is likely that diffusion of NO dominants (19). This interaction of tubules and vessels in the outer medulla by free radicals, called "tubulo-vascular cross-talk", contributes to the modification of the direct vasaconstrictive action of Ang II as seen in the in vivo studies discussed above. Both superoxide production by Ang II stimulation in endothelium of vasa recta and epithelial cells of mTAL was inhibited by pre-incubation with apocynin and diphenyleneiodonium, an inhibitor of $\mathrm{NAD}(\mathrm{P}) \mathrm{H}$ oxidase and flavoprotein, respectively, indicating that the production of superoxide was through NAD(P)H oxidase (19). The receptors involved in these responses remain uncertain. Although both vasa recta and mTAL express AT1 receptors, AT2 receptors were expressed in vasa recta but there is little evidence of AT2 receptors within mTAL as determined with immunohistochemistry in the tissue sections and RT-PCR from isolated microdissected segments (20). Further studies will be required to determine how these receptors are involved in producing $\mathrm{NO}$ and superoxide for the regulation of MBF.

\section{Ang II-mediated medullary circulation in Dahl salt- sensitive rats and renal injury}

Ang II and NO interactions were determined in renal medulla of Dahl salt-sensitive hypertensive (DahlS) rats (12). NO production in response to a subpressor dose of Ang II $\left(5 \mathrm{ng} \cdot \mathrm{kg}^{-1} \cdot \mathrm{min}^{-1}\right)$ using the oxyhemoglobin trapping microdialysis techinique was impaired in low salt fed anesthetized DahlS rats and reduced MBF, while the same dose of Ang II did not alter MBF in BrownNorway (BN) rats (12). Long term (7 days) intravenous administration of Ang II $\left(3 \mathrm{ng} \cdot \mathrm{kg}^{-1} \cdot \mathrm{min}^{-1}\right)$, at a dose of which did not increase blood pressure in $\mathrm{BN}$ rats, caused sustained hypertension. However, when the NO substrate L-arginine was infused into the renal medullary region of DahlS rats, the same dose of Ang II failed to show sustained increase in blood pressure, indicating that reduced NO production by Ang II was responsible for hypertension in DahlS rats (12). Expression of NOS mRNA and protein were also found to be decreased in the outer medulla of DahlS rats. NOS activity in the outer medullary region of DahlS rats determined with an L-arginine to L-citrulline HPLC conversion assay was significantly reduced compared to that of BN rats (12). Moreover, Miyata et al. have shown that L-arginine infused into the renal medullary interstitial space restored the reduction of $\mathrm{MBF}$ and inhibited the increase in blood pressure after a high salt diet in DahlS rats (21).

For oxidative stress, Meng et al. have shown using lucigenin chemiluminescence that DahlS rats fed with high salt diet have increased renal superoxide levels in both renal cortex and medulla (22). Intravenous infusion of the SOD mimetic TEMPOL for 3 weeks to DahlS rats reduced renal superoxide level either with rats infused low or high Na diet (23). In high Na infused DahlS rats, blood pressure was reduced and glomeruloscrerosis and urinary protein excretion were decreased (23). These results indicate that renal oxidative stress is responsible for hypertensive renal injury in DahlS rats. However, as also indicated above, the role of oxidative stress in the regulation of MBF remains to be investigated.

As described above, interstitial $\mathrm{PO}_{2}$ of outer medulla is less than $40 \%$, while tubules in this region such as mTAL is oxygen-dependent due to high levels of metabolic activity required to transport $\mathrm{NaCl}$ (2). Since it has also been shown that this transport is enhanced in DahlS rats compared to that of Dahl salt-resistant rats (24), the greater levels of oxygen consumption with reduced levels of medullary flow could lead to chronic hypoxia. These conditions could explain the vulnerability to renal injury in this region and why interstitial and capillary injury was first found in the outer medullary region as shown by Johnson et al. (25).

\section{Ang II-induced renal injury in renal medullary region and interactions with renal perfusion pressure}

Ang II induces multiple forms of injury in the kidney such as vascular stenosis, glomerular screrosis, tubular necrosis, and interstitial fibrosis (26). Since Ang II increases blood pressure, pressure-dependent and -independent renal injury was determined in Ang II intravenously infused rats with an implanted aortic occluder that enabled chronic servo-control of the perfusion pressure to the left kidney that could be maintained at a normal pressure during the development of hypertension (27). Pressure-induced renal injury was determined by comparison of the right kidney to the left one, whereas effect of circulating Ang II independent of renal perfusion pressure to the renal injury was determined with the comparison of the left kidney to that of sham rats. The results demonstrated that the increase of renal perfusion pressure accounted for nearly $85 \%$ of the medullary injury found in this model of hypertension. This was 
found to be the case for injury in the interlobular arteries, juxtamedullary glomeruli, medullary tubules, and medullary interstitium. However, pressure-independent injury still remained in these regions and was particularly dominant in superficial glomeruli, indicating the direct effects of Ang II within the cortex (27). TGF $-\beta$ and NF- $\kappa$ B were expressed in the pressureinduced interstitial fibrosis, indicating that oxidative stress was involved in the pressure-induced medullary renal injury (27).

\section{Conclusion}

In summary, a number of studies have now demonstrated the physiological functions of renal medullary flow and role of Ang II. Over the past decade, it has also been shown that reactive oxygen and nitrogen species can participate importantly in the regulation of $\mathrm{MBF}$ and in the long-term regulation of arterial blood pressure. The vulnerability of the renal medulla to pressure-induced injury in hypertension has been clearly demonstrated in the rat. However, these events remain to be demonstrated in human subjects, a task that will be challenging given the limited techniques available to study medullary function in human subjects. Although AT1-receptor blockers and ACE inhibitors are now widely used for protection against renal injury in hypertensive and diabetic patients, the functional aspects of the renal medullary function and injury to this region remains unclear and needs to be investigated.

\section{Acknowledgments}

We thank Meredith M. Skelton for careful review of the manuscript. The above studies were mostly carried out at the Medical College of Wisconsin.

\section{References}

1 Cowley AW Jr. Role of the renal medulla in volume and arterial pressure regulation. Am J Physiol. 1997;273:R1-R15.

2 Cowley AW Jr, Mori T, Mattson D, Zou AP. Role of renal NO production in the regulation of medullary blood flow. Am J Physiol. 2003;284:R1355-R1369.

3 Mattson DL, Roman RJ, Cowley AW Jr. Role of nitric oxide in renal papillary blood flow and sodium excretion. Hypertension. 1992;19:766-769.

4 Nakanishi K, Mattson DL, Cowley AW Jr. Role of renal medullary blood flow in the development of L-NAME hypertension in rats. Am J Physiol. 1995;268:R317-R323.

5 Mattson DL, Lu S, Roman RJ, Cowley AW Jr. Relationship between renal perfusion pressure and blood flow in different regions of the kidney. Am J Physiol. 1993;264:R578-R583.

6 Zou AP, Wu F, Cowley AW Jr. Protective effect of angiotensin
II-induced increase in nitric oxide in the renal medullary circulation. Hypertension. 1998;31:271-276.

7 Szentivanyi M Jr, Maeda CY, Cowley AW Jr. Local renal medullary L-NAME infusion enhances the effect of long-term angiotensin II treatment. Hypertension. 1999;33:440-445.

8 Zou AP, Li N, Cowley AW Jr. Production and actions of superoxide in the renal medulla. Hypertension. 2001;37:547553.

9 Makino A, Skelton MM, Zou AP, Roman RJ, Cowley AW Jr. Increased renal medullary oxidative stress produces hypertension. Hypertension. 2002;39:667-672.

10 Chen YF, Cowley AW Jr, Zou AP. Increased $\mathrm{H}_{2} \mathrm{O}_{2}$ counteracts the vasodilator and natriuretic effects of superoxide dismutation by tempol in renal medulla. Am J Physiol Regul Integr Comp Physiol. 2003;285:R827-R833.

11 Makino A, Skelton MM, Zou AP, Cowley AW Jr. Increased renal medullary $\mathrm{H}_{2} \mathrm{O}_{2}$ leads to hypertension. Hypertension. 2003;42:25-30.

12 Szentivanyi M Jr, Zou AP, Mattson DL, Soares P, Moreno C, Roman RJ, et al. Renal medullary nitric oxide deficit of Dahl S rats enhances hypertensive actions of angiotensin II. Am J Physiol. 2002;283:R266-R272.

13 Park F, Mattson DL, Roberts LA, Cowley AW Jr. Evidence for the presence of smooth muscle alpha-actin within pericytes of the renal medulla. Am J Physiol. 1997;273:R1742-R1748.

14 Pallone TL, Zhang Z, Rhinehart K. Physiology of the renal medullary microcirculation. Am J Physiol Renal Physiol. 2003;284:F253-F266.

15 Guyton AC, Coleman TG, Cowley AV Jr, Scheel KW, Manning RD Jr, Norman RA Jr. Arterial pressure regulation. Overriding dominance of the kidneys in long-term regulation and in hypertension. Am J Med. 1972;52:584-594.

16 Cowley AW, Roman RJ, Fenoy FJ, Mattson DL. Effect of renal medullary circulation on arterial pressure. J Hypertens Suppl. 1992;10:S187-S193.

17 Garcia-Estan J, Roman RJ. Role of renal interstitial hydrostatic pressure in the pressure diuresis response. Am J Physiol. 1989; 256:F63-F70.

18 Dickhout JG, Mori T, Cowley AW Jr. Tubulo-vascular nitric oxide cross-talk buffers Ang II-induced medullary vasoconstriction. Circ Res. 2002;91:487-493.

19 Mori T, Cowley AW Jr. Angiotensin II-NAD(P)H oxidase stimulated suproxide modifies tubulo-vascular nitric oxide cross-talk in renal outer medulla. Hypertension. 2003;42:588 593.

20 Miyata N, Park F, Li XF, Cowley AW Jr. Distribution of angiotensin AT1 and AT2 receptor subtypes in the rat kidney. Am J Physiol. 1999;277:F437-F446.

21 Miyata N, Cowley AW Jr. Renal intramedullary infusion of Larginine prevents reduction of medullary blood flow and hypertension in Dahl salt-sensitive rats. Hypertension. 1999;33: 446-450.

22 Meng S, Roberts LJ 2nd, Cason GW, Curry TS, Manning RD Jr. Superoxide dismutase and oxidative stress in Dahl salt-sensitive and -resistant rats. Am J Physiol Regul Integr Comp Physiol. 2002;283:R732-R738.

23 Meng S, Cason GW, Gannon AW, Racusen LC, Manning RD Jr. Oxidative stress in Dahl salt-sensitive hypertension. Hypertension. 2003;41:1346-1352.

24 Ito O, Roman RJ. Role of 20-HETE in elevating chloride 
transport in the thick ascending limb of Dahl SS/Jr rats. Hypertension. 1999;33:419-423.

25 Johnson RJ, Gordon KL, Giachelli C, Kurth T, Skelton MM, Cowley AW Jr. Tubulointerstitial injury and loss of nitric oxide synthases parallel the development of hypertension in the Dahl-SS rat. J Hypertens. 2000;18:1497-1505.
26 Johnson RJ, Alpers CE, Yoshimura A, Lombardi D, Pritzl P, Floege $\mathrm{J}$, et al. Renal injury from angiotensin II-mediated hypertension. Hypertension. 1992;19:464-474.

27 Mori T, Cowley AW Jr. Role of pressure in angiotensin IIinduced renal injury: chronic servo-control of renal perfusion pressure. Hypertension. 2004;43:752-759. 